\title{
INCLUSION STUDIES ON $\alpha$-CYCLODEXTRIN COMPLEXES OF GLIPIZIDE AND GLICLAZIDE WITH EFFECT OF PH
}

\author{
LIZY ROSELET S, PREMA KUMARI J* \\ Department of Chemistry and Research Centre, Scott Christian College (Autonomous), Nagercoil - 629 003, Kanniyakumari, Tamil Nadu, \\ India. Email: premarussel@rediffmail.com
}

Received: 12 September 2016, Revised and Accepted: 7 October 2016

\begin{abstract}
Objective: To synthesize inclusion complexes of oral antidiabetic drugs, i.e., Glipizide (GP) and Gliclazide (Glc) with a-cyclodextrin ( $\alpha$-CD) and varying the $\mathrm{pH}$ which will enhance the solubility and thereby oral bioavailability of the drugs.

Methods: Liquid inclusion complexes were prepared and characterized by ultraviolet-visible (UV-VIS) spectroscopic studies, and fluorescent studies solid inclusion complexes of GP and Glc with $\alpha$-CD were prepared by co-evaporation method and characterized by differential scanning calorimetric studies (DSC) and ${ }^{1} \mathrm{H}$ nuclear magnetic resonance spectroscopic studies ( $\left.{ }^{1} \mathrm{H} \mathrm{NMR}\right)$.
\end{abstract}

Results: The inclusion complexes of both GP and Glc with $\alpha$-CD showed high binding constant values for pH 2 from the UV-VIS spectroscopy studies and fluorescent studies. DSC and ${ }^{1} \mathrm{H}$ NMR spectroscopic studies confirmed the inclusion of GP and Glc inside the $\alpha$-CD cavity.

Conclusion: Formed with more stable inclusion complexes, with $\alpha$-CD are GP and Glc at pH 2, along with pH modulation could be a suitable strategy to enhance the solubility and oral bioavailability of both the drugs.

Keywords: Solubility, Bioavailability, Glipizide, Gliclazide, Inclusion complexes, $\alpha$-Cyclodextrin.

(C) 2017 The Authors. Published by Innovare Academic Sciences Pvt Ltd. This is an open access article under the CC BY license (http://creativecommons. org/licenses/by/4. 0/) DOI: http://dx.doi.org/10.22159/ajpcr.2017.v10i1.15164

\section{INTRODUCTION}

Poor solubility and oral bioavailability are the two great challenges in the pharmaceutical industry. Poor aqueous solubility hinders the in vivo efficacy of the drugs due to low bioavailability, abnormal pharma kinetic profile, intersubject, interspecies variation [1] and thus poses challenging problems in the oral formulation development process.

Glipizide (GP), IUPAC name is 1-cyclohexyl-3-[[P-[2-(5methylpyrazinecarboxamido) ethyl] phenyl]-sulfonyl] urea, Gliclazide (Glc) IUPAC name is 1-[4methylbenzene)sulfonyl]-3octahydrocyclopenta[C] pyrrol-2-yl] urea.

GP and Glc are both second generation sulfonylurea hyperglycemic agents which are widely used in the treatment of noninsulin-dependent diabetes mellitus [2]. Both GP and Glc have very poor aqueous solubility and hence low bioavailability. Consequently, several studies have been developed for enhancement of solubility and bioavailability of GP and Glc through cyclodextrin (CD) inclusion complexes [3-7].

Enhancement in the solubility and the dissolution rate of such drugs can improve the oral bioavailability, which further improves the therapeutic efficacy and patient compliance. Different techniques have been used to improve the solubility and dissolution rate of the poorly soluble drugs, and in middle of the alternatives, the use of CD complexation presented a great interest [8] in recent years. CDs are cyclic oligosaccharides composed of glucopyranose units and can be represented as a truncated cone structure with a hydrophobic cavity. The hydrophobic cavity forms inclusion compounds with a wide range of guest molecules. Inclusion complex formation will modify the physicochemical properties such as solubility, stability, and bioavailability of poorly water-soluble drugs [9].

It is also a well-known fact that the $\mathrm{pH}$ of the medium is one of the important factors affecting the solubility of the drugs [10]. Hence, the present study is to investigate the effect of $\mathrm{pH}$, in selected CD, i.e., $\alpha$-CD complexation on the solubility of GP and Glc. The solubility study is carried out in different media, i.e., $\mathrm{pH} 2$ and 7 to identify the physiological $\mathrm{pH}$ at which the drugs have the highest solubility and also the influence of $\alpha$-CD complexation in enhancing the solubility of GP and Glc.

\section{METHODS}

\section{Reagents and materials}

Analytical grade of GP, Glc, and $\alpha$-CD from Sigma-Aldrich were used for the studies. The solvents used were of analytical grade. Triply distilled water was used for the preparation of stock solutions.

\section{Instruments}

i. Systronics Smart Double beam Spectrophotometer-2203

ii. JASCO Spectrofluorometer FP-8200

iii. NETZSCH DSC 204 Calorimeter

iv. Bruker $300 \mathrm{MHz}$ FT NMR Spectrometer.

\section{Preparation of liquid inclusion complexes}

About 0.0089 of GP was accurately weighed and dissolved in $10 \mathrm{ml}$ solution maintained at $\mathrm{pH} 2$ and 7. Similarly, $0.0064 \mathrm{~g}$ of Glc was weighed and dissolved in $10 \mathrm{ml}$ solution maintained at $\mathrm{pH} 2$ and $\mathrm{pH} 7$. About $0.2918 \mathrm{~g}$ of $\alpha$-CD was weighed and dissolved in $30 \mathrm{ml}$ distilled water. Inclusion complexes were prepared by varying the concentration of $\alpha$-CD from $2 \times 10^{-3} \mathrm{M}$ to $1 \times 10^{-2} \mathrm{M}$ with both the drugs. The reactions were carried out at room temperature $(303 \mathrm{~K})$.

\section{Preparation of solid inclusion complexes}

Solid inclusion complexes of GP and Glc with $\alpha$-CD were prepared by co-evaporation method.

\section{Differential scanning calorimetry (DSC)}

DSC measurements were performed on an NETZSCH DSC 204 calorimeter. Samples of 1-4 mg were accurately weighed in aluminum pans. These samples were then heated over a range of $25^{\circ} \mathrm{C}-300^{\circ} \mathrm{C}$ at a 
constant rate of $10^{\circ} \mathrm{C} /$ minutes in a nitrogen purge of $50 \mathrm{ml} /$ minutes. An empty aluminum pan was used as reference.

\section{${ }^{1} \mathrm{H}$ nuclear magnetic resonance $\left({ }^{1} \mathrm{H}-\mathrm{NMR}\right)$ spectroscopy}

${ }^{1} \mathrm{H}-\mathrm{NMR}$ spectroscopy studies were carried out using Bruker $300 \mathrm{MHz}$ FT NMR Spectrometer. Chemical shifts were reported in ppm $(\delta)$ downfield from tetramethylsilane (internal reference $0 \mathrm{ppm}$ ). Samples were prepared by dissolving in dimethyl sulphoxide.

\section{RESULTS AND DISCUSSION}

\section{Effect of pH}

Absorption studies

From Fig. 2a, it was observed that at $\mathrm{pH} 2$, the absorption spectrum of GP was obtained at $230.8 \mathrm{~nm}$ in the absence of $\alpha$-CD. After the addition of $\alpha-C D$, there is a bathochromic shift from $\lambda_{\max } 230.8$ to $241.3 \mathrm{~nm}$. The absorption spectrum showed a hypsochromic shift from $\lambda_{\max } 235.5$ to $229.5 \mathrm{~nm}$ as depicted in Fig. $2 \mathrm{~b}$. The spectral shifts were characteristic of the inclusion complexes formed by the encapsulation of the GP drug within the $\alpha$-CD cavity.
For Glc, the absorption spectrum showed a bathochromic shift of $\lambda_{\text {ma }}$ 225.9-235.7 $\mathrm{nm}$ indicating the inclusion complex formation at $\mathrm{pH} 2$ as shown in Fig. 3a. A hypsochromic shift was observed with a $\lambda$ from 228.7 to $218.5 \mathrm{~nm}$ at $\mathrm{pH} 7$ shown in Fig. 3b. The spectral shifts indicated the inclusion complex formation.

From Table 1, it was found that as the $\mathrm{pH}$ increases the intensity of absorption increases. The wavelength was shifted hypsochromically. After the addition of $\alpha-C D$, a bathochromic shift was observed at $\mathrm{pH} 2$, and a hypsochromic shift was observed at $\mathrm{pH}$ 7. This shows that as the $\mathrm{pH}$ of the medium increases, the solubility of both the drugs GP and Glc decreases.

\section{Fluorescent studies}

The fluorescent spectrum for GP $\alpha$-CD complex exhibited a bathochromic shift of $\lambda_{\text {max }}$ 348- $371 \mathrm{~nm}$ at $\mathrm{pH} 2$ shown in Fig. $4 \mathrm{a}$. As the $\mathrm{pH}$ was increased to 7 , a hypsochromic shift was observed from $\lambda_{\max } 387$ to $369 \mathrm{~nm}$ as shown in Fig. 4b. The spectral shifts were indicative of encapsulation of the GP within the $\alpha$-CD cavity leading to the formation of inclusion complexes.

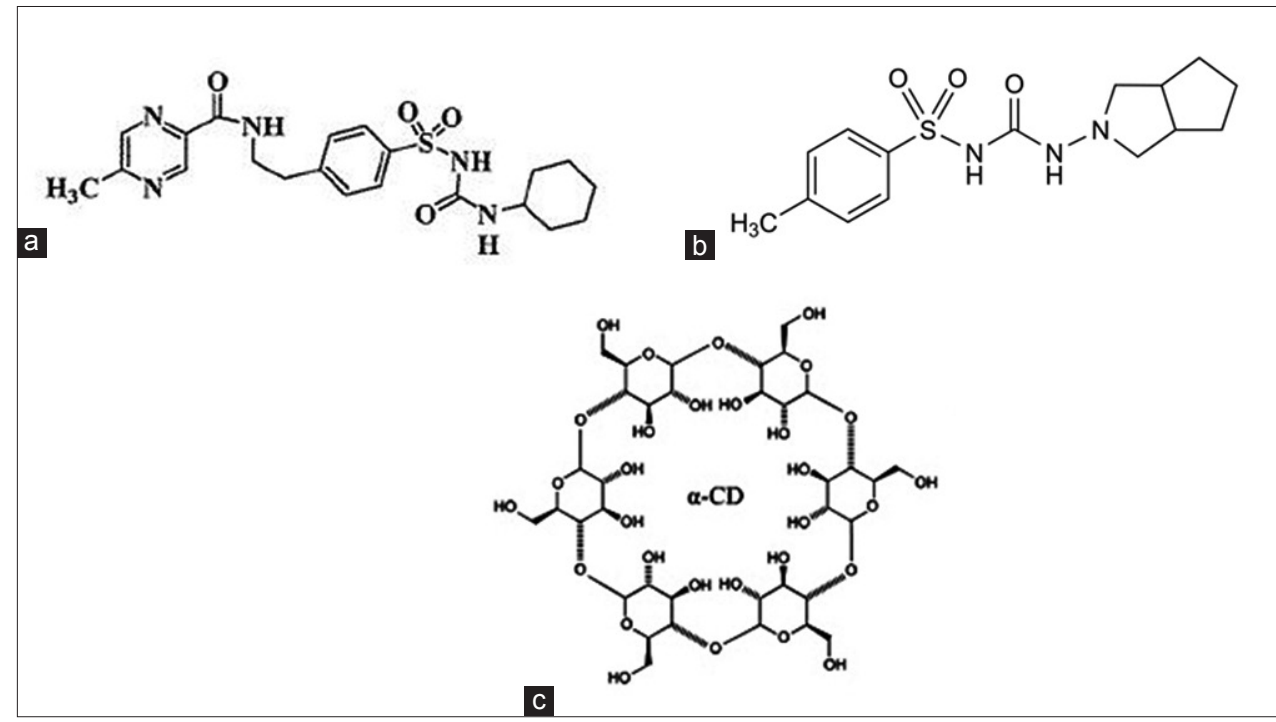

Fig. 1: Structure of (a) Glipizide (b) Gliclazide and (c) $\alpha$-cyclodextrin

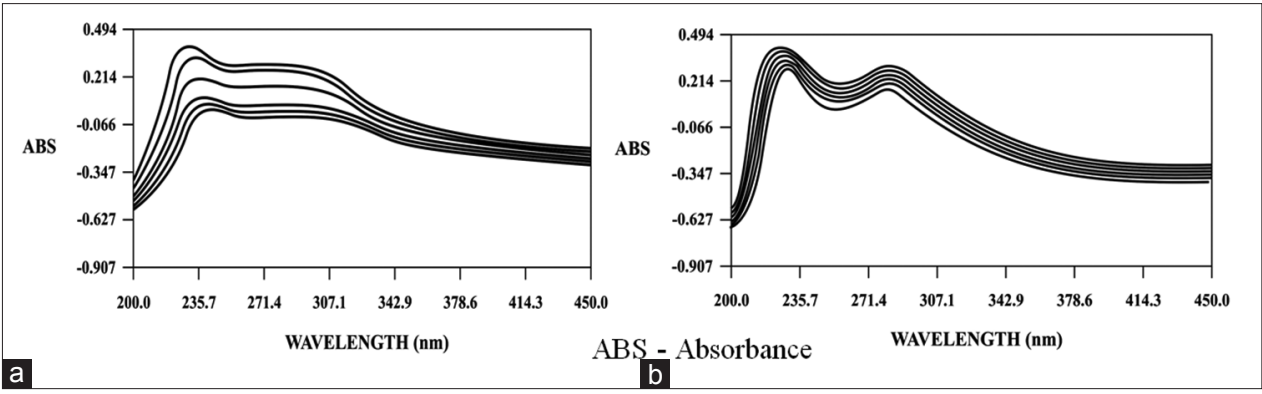

Fig. 2: Absorption spectrum of Glipizide- $\alpha$-cyclodextrin at (a) pH 2 and (b) pH 7. ABS: Absorbance

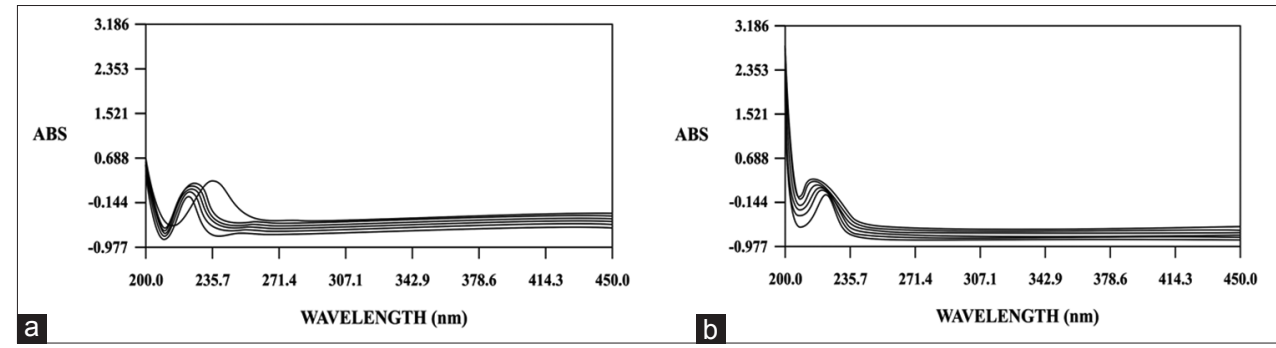

Fig. 3: Absorption spectrum of Gliclazide- $\alpha$-cyclodextrin at (a) pH 2 and (b) pH 7 
For Glc, a bathochromic shift was observed at $\mathrm{pH} 2$ from $\lambda_{\text {max }} 393$ to $407 \mathrm{~nm}$ shown in Fig. 5a. At pH 7, a bathochromic shift was observed from $\lambda_{\max } 388$ to $392 \mathrm{~nm}$ shown in Fig. 5b. The spectral shifts were characteristic of the inclusion complex formation between Glc and $\alpha$-CD.

From Table 2, it was observed that, as the $\mathrm{pH}$ increases the intensity of emission decreases, the wavelength was shifted hypsochromically for GP $\alpha$-CD complexes. For Glc $\alpha$-CD complex, a bathochromic shift was observed with decrease in intensity. This depicted that as the $\mathrm{pH}$ of the medium increases the solubility of both GP and Glc decreases.

The $\alpha$-CD dependence of GP on absorption can be analyzed by the Benesi-Hildebrand plot as given by [11]:

$\frac{1}{A-A_{0}}=\frac{1}{A^{\prime}-A_{0}}+\frac{1}{K_{B}\left(A^{\prime}-A_{0}\right)[\alpha-C D]}$

Where, $[\alpha-C D]$ represents the concentration of $\alpha-C D$ and $A_{0}, A$ are the absorbances in the absence and presence of $\alpha-C D$, respectively, and $K_{B}$ is the binding constant.
From Fig 6a and 7a, it was observed that a reciprocal graph of $\frac{1}{A-A_{0}}$ versus $\frac{1}{[\alpha-C D]}$ yielded a straight line which indicated the formation of GP- $\alpha$-CD inclusion complex with 1:1 and 1:2 stoichiometry. A reciprocal graph of $\frac{1}{A-A_{0}}$ versus $\frac{1}{[\alpha-C D]^{2}}$ yielded a curve which indicated the formation of Glc- $\alpha-\mathrm{CD}$ inclusion complex with 1:2 stoichiometry.

Similarly, the $\alpha$-CD dependence on the fluorescent spectra of GP could be analyzed by the Benesi-Hildebrand plot as given by:

$$
\frac{1}{I-I_{0}}=\frac{1}{I^{\prime}-I_{0}}+\frac{1}{K_{B}\left(I^{\prime}-I_{0}\right)[\alpha-C D]}
$$

Where, $[\alpha-\mathrm{CD}]$ represents the concentration of $\alpha-\mathrm{CD}$ and $I_{\sigma^{\prime}} I$ are the intensities of fluorescence in the absence and presence of $\alpha-\mathrm{CD}$, respectively. From Fig $6 \mathrm{~b}$ and $7 \mathrm{~b}$, it could be inferred that a reciprocal graph of $\frac{1}{I-I_{0}}$ versus $\frac{1}{[\alpha-C D]}$ yielded a straight line which indicated the formation of GP- $\alpha-\mathrm{CD}$ inclusion complex with 1:1 and 1:2 stoichiometry.

Table 1: The absorption spectral data of Glipizide and Gliclazide inclusion complexes at pH 2 and 7

\begin{tabular}{|c|c|c|c|c|c|c|c|c|c|c|}
\hline \multirow[t]{2}{*}{ Drugs } & \multicolumn{5}{|l|}{ pH 2} & \multicolumn{5}{|l|}{ pH 7} \\
\hline & Concn. of $\alpha$-CD & $\lambda_{\mathrm{abs}}$ & Abs & $\frac{1}{A-A_{0}}$ & $\begin{array}{l}\log \\
\varepsilon\end{array}$ & $\lambda_{\text {abs }}$ & Abs & $\frac{1}{A-A_{0}}$ & $\log \varepsilon$ & $1 /[\alpha-C D]$ \\
\hline \multirow[t]{6}{*}{ Glipizide } & 0 & 230.8 & 0.430 & & 4.04 & 235.5 & 0.302 & & 3.88 & \\
\hline & 0.002 & 235.0 & 0.273 & 6.36 & 3.84 & 234.5 & 0.335 & 30.30 & 3.93 & 500 \\
\hline & 0.004 & 236.4 & 0.209 & 4.52 & 3.72 & 233.5 & 0.343 & 24.39 & 3.94 & 250 \\
\hline & 0.006 & 237.1 & 0.155 & 3.63 & 3.59 & 232.5 & 0.379 & 12.98 & 3.98 & 166.6 \\
\hline & 0.008 & 239.3 & 0.117 & 3.19 & 3.47 & 231.5 & 0.385 & 12.04 & 3.99 & 125 \\
\hline & 0.010 & 241.3 & 0.055 & 2.66 & 3.14 & 229.5 & 0.404 & 9.8 & 4.01 & 100 \\
\hline \multirow[t]{6}{*}{ Gliclazide } & 0 & 225.9 & 0.103 & & 3.43 & 228.7 & 0.096 & & 3.40 & \\
\hline & 0.002 & 227.3 & 0.132 & 34.48 & 3.54 & 226.6 & 0.132 & 27.7 & 3.54 & 500 \\
\hline & 0.004 & 228.9 & 0.155 & 19.23 & 3.61 & 223.4 & 0.146 & 20.00 & 3.58 & 250 \\
\hline & 0.006 & 229.1 & 0.164 & 16.39 & 3.63 & 221.0 & 0.171 & 13.33 & 3.65 & 166.6 \\
\hline & 0.008 & 232.0 & 0.187 & 11.90 & 3.69 & 219.7 & 0.209 & 8.84 & 3.74 & 125 \\
\hline & 0.10 & 235.7 & 0.262 & 6.289 & 3.83 & 218.5 & 0.226 & 7.69 & 3.77 & 100 \\
\hline
\end{tabular}

Concn.: Concentration, Abs: Absorbance, Int: Intensity. A : Absorbance of pure Glipizide/Gliclazide, A: Absorbance after addition of $\alpha$-CD, [ $\alpha$-CD]: Concentration of a-CD, CD: Cyclodextrin

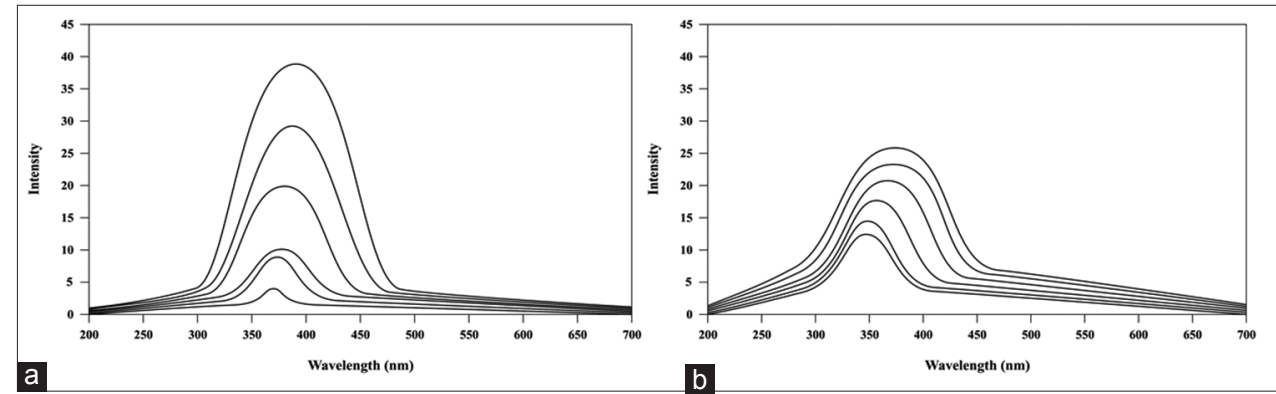

Fig. 4: Fluorescent spectrum of Glipizide- $\alpha$-cyclodextrin at (a) pH 2 and (b) pH 7

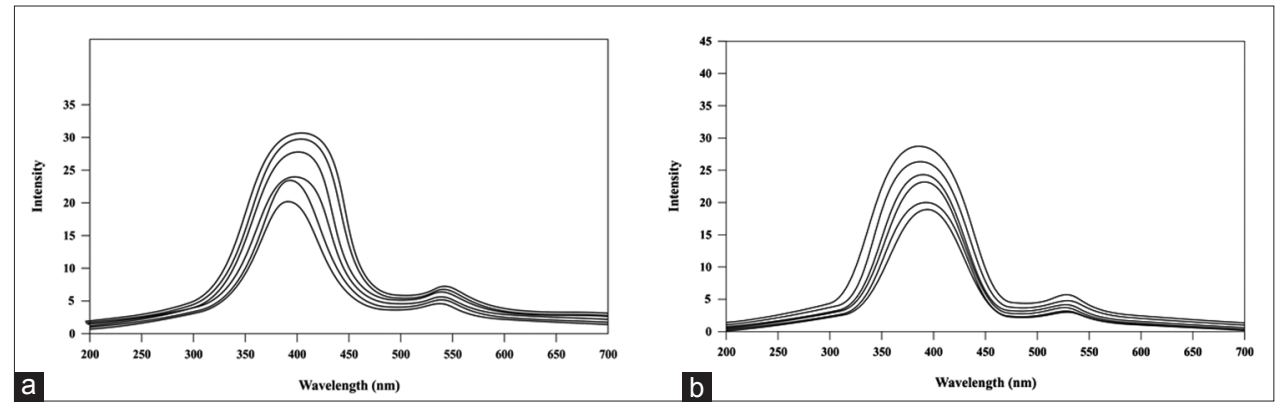

Fig. 5: Fluorescent spectrum of Gliclazide- $\alpha$-cyclodextrin at (a) pH 2 and (b) pH 7 
Table 2: The fluorescent spectral data of Glipizide and Gliclazide inclusion complexes at pH 2 and pH 7

\begin{tabular}{|c|c|c|c|c|c|c|c|c|}
\hline \multirow[t]{3}{*}{ Drugs } & \multicolumn{4}{|l|}{ pH 2} & \multicolumn{4}{|c|}{ pH 7} \\
\hline & Concn. of $\alpha-C D$ & $\lambda_{\text {flu }}$ & Int. & 1 & $\lambda_{\text {flu }}$ & Int. & 1 & $1 /[\alpha-C D]$ \\
\hline & & & & $\overline{I-I_{0}}$ & & & $\overline{I-I_{0}}$ & \\
\hline \multirow[t]{5}{*}{ Glipizide } & 0 & 348 & 12.46 & & 387 & 38.64 & & \\
\hline & 0.002 & 349 & 14.54 & 0.480 & 385 & 28.32 & 0.0968 & 500 \\
\hline & 0.004 & 354 & 17.08 & 0.216 & 380 & 19.14 & 0.0534 & 250 \\
\hline & 0.006 & 361 & 21.11 & 0.115 & 378 & 10.15 & 0.0351 & 166.6 \\
\hline & 0.010 & 371 & 25.7 & 0.071 & 369 & 4.2 & 0.0290 & 100 \\
\hline \multirow[t]{6}{*}{ Gliclazide } & 0 & 393 & 20.03 & & 388 & 29.4 & & \\
\hline & 0.002 & 395 & 23.7 & 0.2724 & 389 & 26.0 & 0.2941 & 500 \\
\hline & 0.004 & 398 & 24.4 & 0.2288 & 390 & 24.7 & 0.2127 & 250 \\
\hline & 0.006 & 401 & 27.8 & 0.1287 & 391 & 22.9 & 0.1538 & 166.6 \\
\hline & 0.008 & 404 & 29.7 & 0.1034 & 392 & 20.1 & 0.1075 & 125 \\
\hline & 0.10 & 407 & 31.0 & 0.0911 & 392 & 18.9 & 0.0952 & 100 \\
\hline
\end{tabular}

$\lambda_{\text {flu }}$ : Wavelength of fluorescence, Int.: Intensity, I0 Intensity of drug without $\alpha$-CD, I: Intensity after addition of a-CD, [ $\alpha$-CD]: Concentration of a-CD, CD: Cyclodextrin

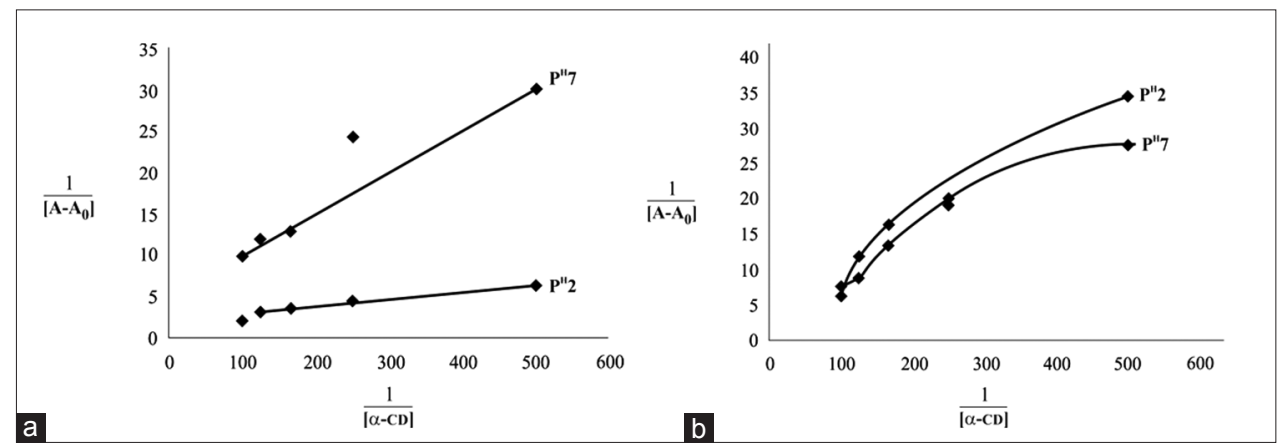

Fig. 6: Benesi Hildebrand plot of (a) Glipizide- $\alpha$-cyclodextrin (CD) at pH 2 and 7 (b) Gliclazide- $\alpha$-CD at pH 2 and pH 7

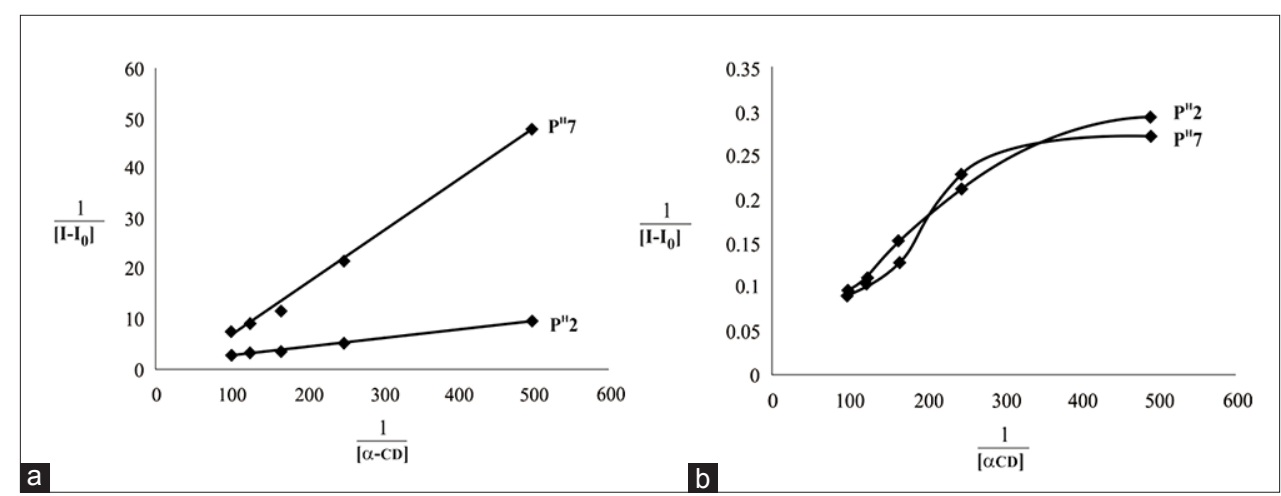

Fig. 7: Benesi Hildebrand plot of (a) Glipizide- $\alpha$-cyclodextrin (CD) at pH 2 and 7 (b) Gliclazide- $\alpha$-CD at pH 2 and pH 7

A reciprocal graph of $\frac{1}{I-I_{0}}$ versus $\frac{1}{[\alpha-C D]^{2}}$ yielded a straight line

which indicated the formation of Glc $\alpha$-CD inclusion complex with 1:2 stoichiometry.

A linear relation is obtained in the case of absorption and fluorescence at both $\mathrm{pH}$ values for GP and Glc which revealed the stoichiometry of the complex formed by the GP with $\alpha$-CD is $1: 1,1: 2$ and that of Glc with $\alpha-C D$ is $1: 2$. Moreover, the linearity relation also indicated the increase in aqueous solubility of the drug upon complexation with increase in the concentration of $\alpha$-CD.

The binding constant for GP $\alpha$-CD complex from absorption studies was found to be $266 / \mathrm{M}$ at pH 2 and $245 / \mathrm{M}$ at pH 7. From fluorescent studies, it was evaluated as $276 / \mathrm{M}$ at pH 2 and $80.97 / \mathrm{M}$ at $\mathrm{pH} 7$.

For Glc $\alpha$-CD complexes, the binding constant was calculated to be $117.5 / \mathrm{M}$ at $\mathrm{pH} 2$ and 85.47 at $\mathrm{pH} 7$ from absorption studies. Fluorescent studies showed that the binding constant was $112.0 / \mathrm{M}$ at $\mathrm{pH} 2$ and 84.1/M at $\mathrm{pH} 7$

As the value of binding constant increases, the stability of the inclusion complex also increases and hence the solubility of the drug increases. It was found that the value of binding constants decreases with increase in $\mathrm{pH}$ from 2 to 7 . So, with increase in $\mathrm{pH}$, the stability of the inclusion complexes formed between GP and Glc with $\alpha-\mathrm{CD}$ and hence the solubility of both GP and Glc decreases.

\section{DSC studies}

DSC analysis was performed to characterize inclusion complexes with $\alpha$-CD by comparing the thermal behavior of the individual components as well as their individual components [12-14].

When the guest molecules are incorporated into the CD cavity or in the crystal lattice, their melting point and boiling point usually shift to different temperatures or disappear within the temperature range 


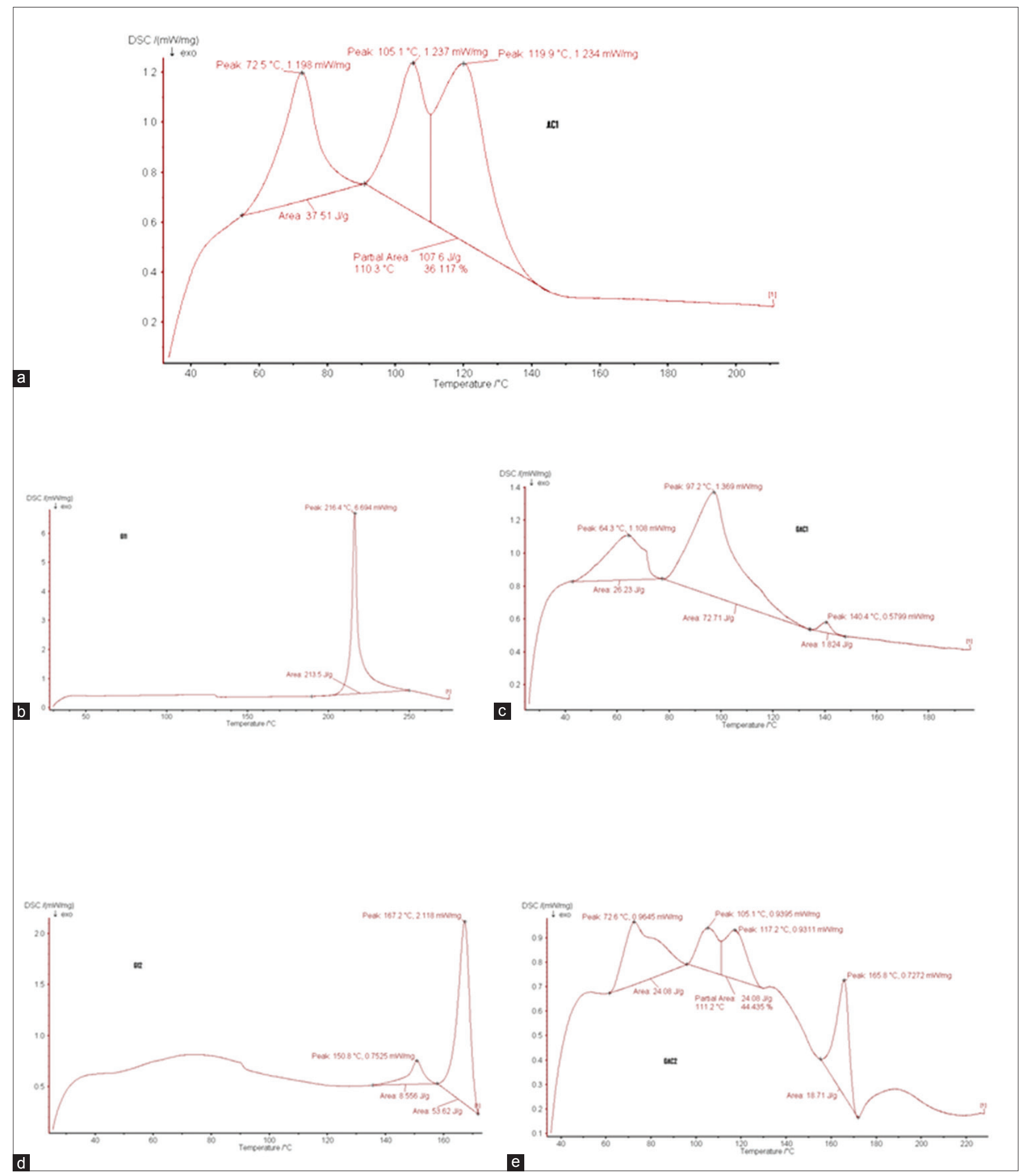

Fig 8: Differential scanning calorimetric thermograms of (a) $\alpha$-cyclodextrin (CD) (b) Glipizide (c) Glipizide $\alpha$-CD (d) Gliclazide and (e) Gliclazide $\alpha$-CD complex

when CD lattice is decomposed. This is one of the best method for detecting drug-CD complexation [15]

The DSC thermograms of $\alpha$-CD showed three exothermic peaks at $72.5^{\circ} \mathrm{C}, 106.1^{\circ} \mathrm{C}$, and $119.9^{\circ} \mathrm{C}$ which correspond to the dehydration of $\alpha-\mathrm{CD}$ (Fig. 8a). Further, the thermogram was broadened indicative of the decomposition of $\alpha$-CD and transition from crystallinity to amorphous nature. The DSC thermogram of GP showed one sharp characteristic exothermic peak at $216.4^{\circ} \mathrm{C}$ and $10.4^{\circ} \mathrm{C}$ corresponding to its melting point (Fig. 8b). However, in the case of the GP $\alpha$-CD thermograms, three exothermic peaks were observed at $64.3^{\circ} \mathrm{C}, 97.2^{\circ} \mathrm{C}$, and $140.4^{\circ} \mathrm{C}$ corresponding to the polymorphs formed during the fusion process via the dehydration of $\alpha$-CD. The peak corresponding to the melting point of GP became extinct or disappeared in the DSC thermogram (Fig. 8c). 
The extinction or disappearance of the exothermic peak of GP in GP $\alpha$-CD thermogram was strong evidence for the total encapsulation of the GP in the $\alpha$-CD cavity. This also suggested the formation of 1:1 stoichiometry of inclusion complex between $\alpha$-CD and GP.

The DSC thermograms of Glc showed a sharp characteristic exothermic peak at $167.2^{\circ} \mathrm{C}$ corresponding to its melting point (Fig. 8d). The broad peak around $40^{\circ} \mathrm{C}-80^{\circ} \mathrm{C}$ is due to the amorphous nature of Glc. The DSC thermogram of Glc $\alpha$-CD complex indicated the characteristic exothermic peaks found in $\alpha$-CD, i.e., at $72.6^{\circ} \mathrm{C}, 105.1^{\circ} \mathrm{C}$, and $117.2^{\circ} \mathrm{C}$ due to the dehydration of $\alpha$-CD (Fig. 8e).

Moreover, there is a shift of the exothermic peak characteristic of Glc from $167.2^{\circ} \mathrm{C}$ to $165.8^{\circ} \mathrm{C}$, toward lower temperature. This is indicative of the partial encapsulation of the Glc drug in the $\alpha$-cyclodextrn cavity. Hence, the inclusion complex formed is not of type 1:1. Instead, the stoichiometry was found to be $1: 2$.

\section{NMR spectroscopic studies}

The most direct evidence for the inclusion of a guest molecule into a CD cavity is obtained by ${ }^{1} \mathrm{H}$-NMR spectroscopy $[16,17]$. ${ }^{1} \mathrm{H}$-NMR may also be used to determine the direction of penetration of guest molecule into the CD cavity $[18,19]$. The $\mathrm{H}-3$ and $\mathrm{H}-5$ protons of $\mathrm{CD}$ which are directed toward the interior of the $\mathrm{CD}$ will show a significant upfield shift if inclusion does indeed occur and the $\mathrm{H}-1, \mathrm{H}-2$, and $\mathrm{H}-4$ protons located at the exterior of the cavity will show marginal upfield shift. The spectrum of the guest molecule may also be changed upon inclusion complex formation $[20,21]$.

The ${ }^{1} \mathrm{H}$-NMR chemical shift for each proton of $\alpha$-CD was evaluated for the formation of inclusion complex between GP and $\alpha$-CD.

The formation of inclusion complexes was studied by the changes in the chemical shifts caused by the guest and the host to each other [22-24]. In the structure of $\alpha-\mathrm{CD}, \mathrm{H}-3$ and $\mathrm{H}-5$ protons are located inside the cavity, H-3, H-5 located near the wider rim, and H-5 located at the narrow rim of the CD cavity. All other protons $\mathrm{H}-1, \mathrm{H}-2$, and $\mathrm{H}-4$ are located on the exterior of the cavity.

From the Table $3, \Delta \delta H-3=\Delta \delta H-5=-0.17<0$, for the GP- $\alpha$-CD complex. This showed that inclusion or encapsulation of GP inside the $\alpha$-CD cavity took place, and an upfield was observed in the inclusion complex due to the dissolution of GP inside the $\alpha$-CD cavity.

Further, the spectrum of pure GP was varied in the case of GP $\alpha$-CD complex. This proved as a strong evidence for the inclusion complex formation.

The observed shielding effect can be attributed to the diamagnetic anisotropy effect due to the inclusion of a group rich in $\pi$ electrons (aromatic ring) into the hydrophobic central cavity of $\alpha$-CD molecule.

From Table 4, it was observed $\Delta \delta \mathrm{H}-3>\Delta \delta \mathrm{H}-5>0$, which indicated encapsulation of the drug Glc in the $\alpha$-CD cavity took place. The upfield shift observed in Glc $\alpha$-CD inclusion complex was due to the dissolution of Glc drug inside the $\alpha$-CD cavity and are shielded by the H-3 and H-5 protons of $\alpha-C D$.

Comparative study of the effect of $\mathrm{pH}$ on Glipizide and Gliclazide $\alpha$-CD inclusion complexes

From the absorption and fluorescent studies, it was observed that the binding constant values were higher at $\mathrm{pH} 2$ for both GP and Glc $\alpha$-CD inclusion complexes. Ionized species were present at $\mathrm{pH} 2$ in both the complexes. However, it may be assumed that the ionic species were sufficiently far away from the hydrophobic cavity of the $\alpha$-CD. The binding constant values of GP and Glc $\alpha$-CD complexes were comparatively lower at $\mathrm{pH}$ 7. This phenomenon was exhibited by both the drugs because of the sulphonylurea group present in both the drug. The other groups do not interfere much with inclusion
Table 3: ${ }^{1} \mathrm{H}$-chemical shifts (ppm) of the protons of $\alpha$-cyclodextrin and Glipizide in the free and inclusion complex states

\begin{tabular}{llll}
\hline Proton $\boldsymbol{\alpha}$-CD & $\boldsymbol{\delta}_{\mathbf{0}}$ (free) $\boldsymbol{\alpha}$-CD & $\begin{array}{l}\boldsymbol{\delta}_{\mathrm{c}} \text { (complex) } \\
\text { Glipizide- } \boldsymbol{\alpha} \text {-CD }\end{array}$ & $\boldsymbol{\Delta} \boldsymbol{\delta}\left(\boldsymbol{\delta}_{\mathrm{c}}-\boldsymbol{\delta}_{\mathbf{0}}\right)$ \\
\hline H-1 & 4.98 & 4.97 & -0.01 \\
H-2 & 3.63 & 3.62 & -0.01 \\
H-3 & 3.82 & 3.99 & -0.17 \\
H-4 & 3.40 & 3.77 & -0.03 \\
H-5 & 3.75 & 3.58 & -0.17 \\
H-6 & 3.92 & 3.89 & -0.03 \\
\hline
\end{tabular}

$\delta_{0}$ : Chemical shift of pure $\alpha-\mathrm{CD}, \delta_{\mathrm{C}}$ : Chemical shift of Glipizide $\alpha$-CD complex, $\Delta \delta$ : Difference in chemical shift between complex and pure a-CD. CD: Cyclodextrin

Table 4: ${ }^{1} \mathrm{H}-\mathrm{NMR}$ chemical shifts (ppm) of the protons of $\alpha$-CD and Gliclazide in the free and inclusion complex states

\begin{tabular}{llll}
\hline Protons $\boldsymbol{\alpha}$-CD & $\boldsymbol{\delta}_{\mathbf{0}}$ (free) $\boldsymbol{\alpha}$-CD & $\boldsymbol{\delta}_{\mathbf{c}}$ Gliclazide $\boldsymbol{\alpha}$-CD & $\boldsymbol{\Delta} \boldsymbol{\delta}=\boldsymbol{\delta}_{\mathbf{c}}-\boldsymbol{\delta}_{\mathbf{0}}$ \\
\hline H-1 & 4.98 & - & - \\
H-2 & 3.63 & 3.27 & -0.36 \\
H-3 & 3.96 & 2.78 & -1.18 \\
H-4 & 3.40 & 2.62 & -0.78 \\
H-5 & 3.78 & 2.47 & -1.31 \\
H-6 & 3.92 & 2.33 & -1.59 \\
\hline
\end{tabular}

$\delta_{0}$ : Chemical shift of pure $\alpha$-CD, $\delta_{\mathrm{C}}$ : Chemical shift of Gliclazide $\alpha$-CD complex

$\Delta \delta$ : Difference in chemical shift between complex and pure $\alpha-C D$. CD: Cyclodextrin

complex formation. Increase in $\mathrm{pH}$ of the medium decreases the stability of the inclusion complexes formed and hence the solubility of both GP and Glc. Therefore, it was evident that the most favorable $\mathrm{pH}$ for increasing the solubility of the drugs was at $\mathrm{pH} 2$ and less favorable $\mathrm{pH}$ was 7 . It was often assured that the unionized species were primarily responsible for the formation of the complex and subsequent increase in solubility. However, ionization of a drug can also result in enhanced complexation, and the solubility of the ionized drug complex can sometimes exceed the solubility of the unionized drug complex $\alpha$-CD plays an important role in the solubility of GP and $\mathrm{Glc}$ together with $\mathrm{pH}$ adjustment. The solubility of the corresponding complexes can be much higher than what could be expected by increasing the total drug solubility through only appropriate $\mathrm{pH}$ adjustment. This is possible due is the specific interaction of the drugs molecules with the hydrogen bond system of the $\alpha-C D$ and/ or the modification of the hydrogen bounding network of the surrounding water molecules, the drugs can be strongly bound inside the $\alpha$-CD cavity, even when they are in ionized form because their basic center, and hence, the charge can be located quite far away from the molecules hydrophobic part that is included in the $\alpha$-CD cavity so that the complexation ability is not significantly compromised.

\section{CONCLUSION}

The absorption and fluorescent spectral studies confirmed the formation of $\alpha$-CD inclusion complexes with both GP and Glc. From absorption and fluorescent studies, stoichiometry of GP $\alpha$-CD complex is 1:1, 1:2 and that of Glc $\alpha$-CD complex is $1: 2 .{ }^{1} \mathrm{H}$ NMR and DSC studies also served as evidence for the formation of inclusion complexes. The soluble and stable complexes were formed at $\mathrm{pH}$ value 2 . The present study has demonstrated the possibility to improve the aqueous solubility of GP and Glc by simultaneous $\alpha$-CD complexation and $\mathrm{pH}$ value modulation. High stability constant values were obtained only at $\mathrm{pH} 2$ rather than $\mathrm{pH} 7$. This has suggested that $\alpha$-CD complexation accompanied with $\mathrm{pH}$ value modulation may be a suitable strategy to enhance the aqueous solubility of both GP and Glc, thus overcoming the problems associated with low bioavailability in the pharmaceutical formulation of both the drugs. 


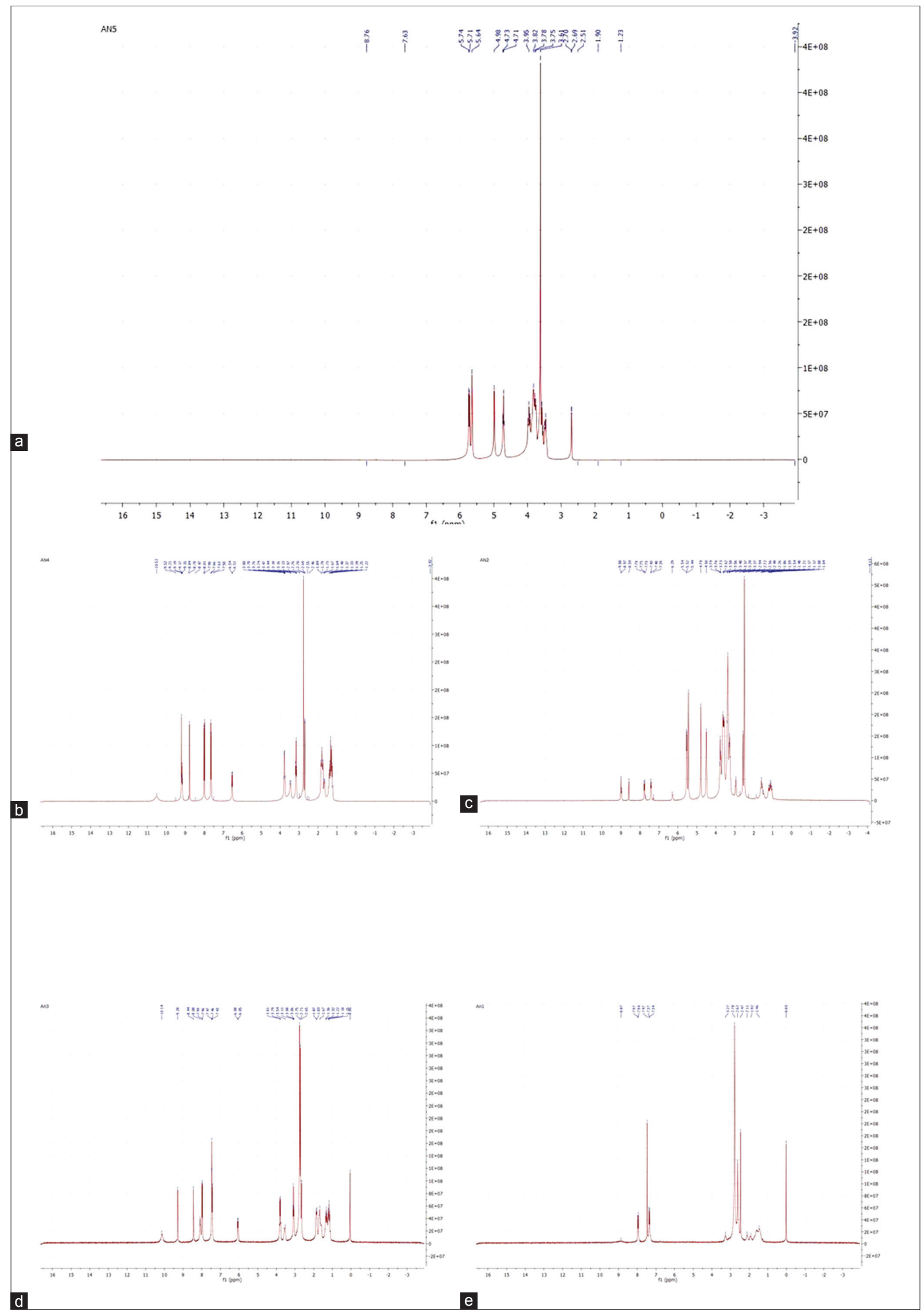

Fig 9: ${ }^{1} \mathrm{H}$-nuclear magnetic resonance spectra of (a) $\alpha$-cyclodextrin (CD) (b) Glipizide (c) Glipizide $\alpha$-CD (d) Gliclazide and (e) Gliclazide $\alpha$-CD complex 


\section{ACKNOWLEDGMENT}

The authors are thankful to the Research Department of Chemistry, Scott Christian College, Nagercoil, for providing necessary facilities.

\section{REFERENCES}

1. van de Waterbeemd $H$. The fundamental variables of the biopharmaceutics classification system (BCS): A commentary. Eur J Pharm Sci 1998;7(1):1-3.

2. Brogden RN, Heel RC, Pakes GE, Speight TM, Avery GS. Glipizide: A review of its pharmacological properties and therapeutic use. Drugs 1979;18(5):329-53.

3. Zhang YM, Li X, Sun CS, Chen CF. The study on the preparation and spectroscopic properties of hydroxypropyl-beta-cyclodextrin/glipizide inclusion complex. Guang Pu Xue Yu Guang Pu Fen Xi 2008;28(3):711-4.

4. Zhang S, Nie SF, Wei LI, Liu HF, Pan WS. Study on inclusive reaction of water-soluble $\beta$-cyclodextrin polymer with glipizide. Chin Pharm J 2007;42:601.

5. Shivakumar HN, Desai BG, Pandya S, Karki SS. Influence of betacyclodextrin complexation on glipizide release from hydroxypropyl methylcellulose matrix tablets. PDA J Pharm Sci Technol 2007;61(6):472-91.

6. Ammar HO, Salama HA, Ghorab M, El-Nahhas SA, Elmotasem H. A transdermal delivery system for glipizide. Curr Drug Deliv 2006;3(3):333-41.

7. Aly AM, Qato MK, Ahmad MO. Enhancement of the dissolution rate and bioavavailability of glipizide through cyclodextrin inclusion complex. Pharm Technol 2003;20:54

8. Riekes MK, Tagliari MP, Granda A, Kumine KG. Dissolution rate of amiodarone by complexation with $\beta$-cyclodextrin through different methods. Mater Sci Eng 2010;30:1008-13.

9. Lu Z, Cheng B, Hu Y, Zhang Y, Zou Y. Complexation and antioxidant activity. Food Chem 2009;113:17-20

10. Amidon GI, Jinno J, Man OD, Crison JR. Dissolution of ionisable water-insoluble drugs; The combined effect of $\mathrm{pH}$ and surfactant. J Pharm Sci 2000;89(82):268-74.

11. Benesi HA, Hildebrand JH. A spectrophotometric investigation of the interaction of iodine with aromatic hydrocarbons. J Am Chem Soc 1949;71(8):2703.

12. Bilensoy E, Cirpanli Y, Dogan AL. Thermosensitive mucoadhesive gel formulation loaded with 5-Fu: Cyclodextrin complex for HPV-induced cervical cancer. J Incl Phenom Macrocycl Chem 2007;57:363-70.

13. Calderini A, Pessine FB. Synthesis and characterization of inclusion complex of the vasodilator drug minoxidel with beta-cyclodextrin. J Incl Phenom Macrocycl Chem 2008;60:369-77.

14. Cevher E, Sensoy D, Zloh M, Mülazimoglu L. Preparation and characterisation of natamycin: Gamma-cyclodextrin inclusion complex and its evaluation in vaginal mucoadhesive formulations. J Pharm Sci 2008;97(10):4319-35.

15. Talegaonkar S, Khan AY, Iqbal Z, Khar RK, Ahmed FJ, Khan ZI. Development and characterization of paracetamol complexes with hydroxypropyl- $\beta$-cyclodextrin. Iran J Pharm Res 2007;6(2):95-9.

16. Jadhav GS, Vavia PR. Physicochemical, in silico and in vivo evaluation of a danazol-beta-cyclodextrin complex. Int J Pharm 2008;352(1-2):5-16.

17. Sinha VR, Anitha R, Ghosh S, Nanda A, Kumria R. Complexation of celecoxib with beta-cyclodextrin: Characterization of the interaction in solution and in solid state. J Pharm Sci 2005;94(3):676-87.

18. Rao BP, Sarasija S, Narendra C. Physiochemical characterization of hydroxypropyl - $\beta$-cyclodextrin complexes of rifampiein for improved anti-tubercular activity and stability. Indian Drugs 2006;43(8):678-82.

19. Semalty A, Tanwar SY. Preparation and characterization of cyclodextrin inclusion complexes for improving solubility and dissolution of nimesulide. World J Pharm Sci 2014;2(1):72-8.

20. Ribeiro LS, Falcão AC, Patrício JA, Ferreira DC, Veiga FJ. Cyclodextrin multicomponent complexation and controlled release delivery strategies to optimize the oral bioavailability of vinpocetine. J Pharm Sci 2007;96(58):2018-28.

21. Duan MS, Zhao N, Ossurardóttir IB, Thorsteinsson T, Loftsson T. Cyclodextrin solubilization of the antibacterial agents triclosan and triclocarban: Formation of aggregates and higher-order complexes. Int J Pharm 2005;297(1-2):213-22.

22. McAlpine SR, Garcia-Garibay MA. Binding studies of adamantanecarboxylic acid and a naphthyl-bound beta-cyclodextrin by variable temperature (1)H NMR. J Org Chem 1996;61(23):8307-9.

23. Bergeron RJ, Channing MA, McGovern KA. Dependence of cycloamylose-substrate binding on charge. J Am Chem Soc 1978; 100:2878-83.

24. Wood DJ, Hruska FE, Sacnger WJ. H-NMR study of the inclusion of aromatic molecules in $\alpha$-cyclodextrin. J Am Chem Soc 1977;99(6):1735-40. 